\title{
Agricultura de precisión: programación del riego en tiempo real
}

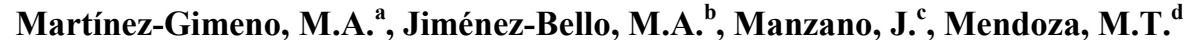

${ }^{a}$ Centro de Edafología y Biología Aplicada del Segura - Consejo Superior de Investigaciones Científicas (CEBAS-CSIC). Campus Universitario de Espinardo, 30100, Murcia.

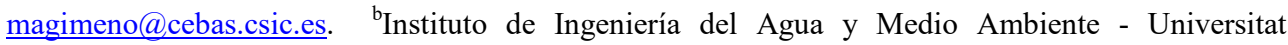
Politècnica de València (IIAMA - UPV). Camino de Vera s/n, 46022, Valencia. mijibar@dihma.upv.es. ${ }^{\circ}$ Departamento de Ingeniería Rural y Agroalimentaria - Universitat Poliècnica de València (DIRA - UPV). Camino de Vera s/n, 46022, Valencia. juamanju@agf.upv.es. ${ }^{\mathrm{d}}$ Departamento de Biología y Geología IES Isabel de Villena (Valencia) e-mail: mendoza_marbal@gva.es

\begin{abstract}
The use of new tools for irrigation planning is an increasingly widespread practice. This step is essential in order to make this practice sustainable, both in the environmental and economical aspects. It is important to provide students the knowledge of these tools and make them competent in its use. An innovative approach has been proposed that integrates the use of a free public web server that guides the student in the calculation of the theoretical plant water needs. Furthermore, this result is associated with the assessment of soil water moisture by means of the probes called Frequency Domain Reflectometry (FDR). This device helps to adjust time and frequency irrigation.

It is emphasized that this innovative methodology does not provide a unique and objective solution, which allows an assessment that is based on a compared and agreed result by groups of students, which encourages collaborative work. In addition, this methodology is aimed at bringing together a range of knowledge to develop capabilities in a real environment. This link with facilitates understanding of the calculation process and increases the interest of the students.
\end{abstract}

Keywords: active methodology, real case, new technologies, integration, applicability, assessment.

\footnotetext{
Resumen

La utilización de las nuevas tecnologías en la planificación del riego es una práctica cada vez más extendida con el fin de hacer esta práctica sostenible tanto en el ámbito medioambiental como en el económico. Es imprescindible transmitir al alumnado la existencia de este tipo de herramientas y hacerles competentes en su uso. Se ha planteado una metodología que integre el uso de un servidor web público y gratuito que orienta al alumno en el cálculo de las necesidades de riego teóricas. Adicionalmente se asocia este resultado al
} 
análisis del contenido de la humedad del suelo mediante el uso de sondas denominadas Frequency Domain Reflectometry (FDR). Este último dispositivo permite ajustar el tiempo de riego y la frecuencia del mismo.

Se destaca que esta innovadora metodología no proporciona un solución única y pero sí objetiva, lo cual permite realizar una evaluación que se basa en un resultado contrastado y consensuado por grupos de alumnos, lo cual impulsa el trabajo colaborativo. Además esta metodología está dirigida a aglutinar una serie de conocimientos en capacidades a desarrollar un ámbito real. Esta vinculación con la práctica directa facilita la visualización del proceso de cálculo y aumenta satisfactoriamente el interés del alumnado.

Palabras clave: metodología activa, caso real, nuevas tecnologías, integración, aplicabilidad, evaluación.

\section{Introducción}

La puesta en práctica de la teoría desarrollada en las clases magistrales no siempre llega a captar la atención del alumnado. Es por ello que es necesario estimular la atención de los alumnos con metodologías actuales y cercanas a la realidad tecnológica actual. Tal y como indicaba Bain (2006), es aconsejable crear las condiciones en las que la mayor parte de nuestros estudiantes, si no todos, conseguirá convertir en realidad su potencial de aprendizaje. De las diversas teorías de aprendizaje, el socio-constructivismo aglutina una serie de actividades que ayudan a conducir al alumno hacia el aprendizaje eficiente, pues contextualizan el objetivo que se trabaja y se vincula a la realidad social que lo rodea. Es importante también destacar la responsabilidad que adquiere el alumno con este tipo de actividades, pues el resultado de su aprendizaje será directamente aplicable y afectará al mundo real. Este enfoque trata de despertar, al menos, la curiosidad hacia un nuevo horizonte del conocimiento. Por todo ello, es evidente que toda actividad que se vincule a una aplicación práctica y directa de las enseñanzas adquiridas, es una transmisión de conocimientos y capacidades más eficiente. En definitiva, las actividades docentes deben estar dirigidas a un aprendizaje significativo.

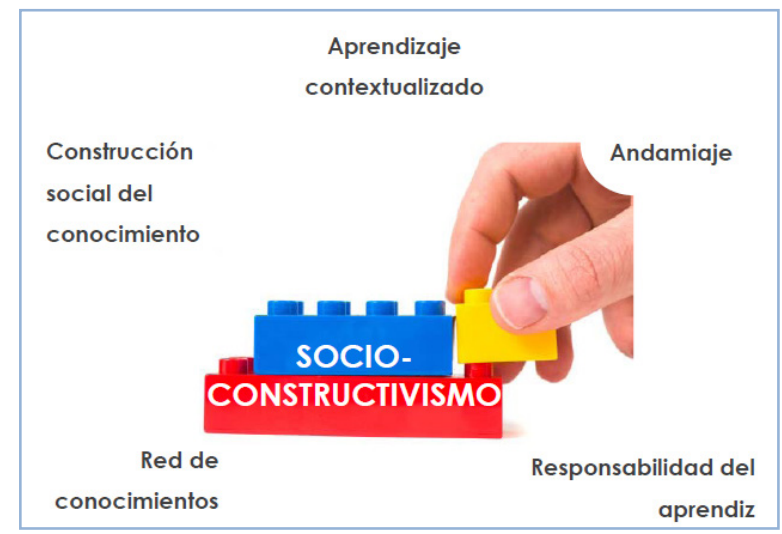

Fig. 1 Esquema conceptual del socio-constructivismo. Fuente: ICE-UPV. Curso PAU.

(cc) EY-NC-ND 2016, Universitat Politècnica de València 
Las nuevas tecnologías aplicadas al ámbito de la agricultura, son una realidad que es preciso transmitir al alumnado, pues son éstas las que permiten abordar los principales problemas a los que se enfrentan productores, técnicos y agricultores en el desempeño de su profesión. El riego es uno de las prácticas más importantes en la agricultura para lograr producciones sostenibles tanto a nivel económico como medioambiental, pues consume entre el $70-80 \%$ de los recursos hídricos disponibles (FAO, 2006). También el consumo energético está usualmente presente en todas las instalaciones de riego a presión (goteo, aspersión y difusión). Éstas requieren equipos de bombeo para proporcionar el agua con suficiente presión con el fin de aportar las cantidades necesarias en los puntos de aplicación en parcela y con una uniformidad adecuada.

En estos dos recursos limitados (agua y energía) es dónde se posiciona el objetivo de este trabajo, desarrollando una metodología de aprendizaje que permita abordar el manejo del riego de forma eficiente. Pero los resultados de aprendizaje de la metodología planteada no sólo buscan la formación del alumno en conceptos sobre el manejo y planificación del riego, sino que pretenden profundizar en el aprendizaje en base a casos reales y conducir al alumno a la toma de decisiones en tiempo real. Por otro lado la aplicación de las nuevas tecnologías requiere un esfuerzo, por parte del alumno, de integración y manejo de diferentes herramientas y fuentes de datos.

\section{Objetivos}

Los objetivos de la metodología planteada abarcan diversas etapas, todas ellas basadas en unos conocimientos teóricos de la asignatura y otras materias en las que se fundamenta y se articulan en este trabajo, y que permitirían al alumno adquirir las competencias necesarias para la planificación eficiente del riego de cultivos. Se pretende diseñar materiales didácticos y metodologías que permitan alcanzar los siguientes resultados de aprendizaje específicos:

- Analizar casos reales con intención de comprender y proponer soluciones aplicables

- Primar el uso de las Nuevas Tecnologías de la Información y Comunicación (TICs)

- Fomentar la motivación intrínseca e impulsar la autonomía de los alumnos

Además esta propuesta de trabajo prestará especial atención a las competencias transversales. Se trata de potenciar la adquisición de un conjunto de habilidades, contextualizadas en el marco de la actividad, que permitirán al alumno afrontar situaciones desde diferentes perspectivas, que no se ciñan únicamente a los contenidos específicos adquiridos. En este caso, se pueden trabajar las siguientes competencias transversales definidas por el Instituto de Ciencias de la Educación (ICE) (http://www.upv.es/entidades/ICE/info/931816normalc.html) de la UPV:

- Compresión e integración

- Aplicación y pensamiento práctico

- Trabajo en equipo y liderazgo 


\section{Desarrollo de la innovación}

La metodología propuesta en el presente trabajo se llevó a cabo por primera vez con los alumnos especialistas en Ingeniería del Riego de la Universidad Tecnológica de Chile Instituto Profesional Centro de Formación Técnica (INACAP) asistentes al curso “Administración y Diseño de Irrigación” organizado por el Centro de Formación Permanente (CPF) de la Universitat Politècnica de València (UPV). Durante el desarrollo del curso, se hizo patente la necesidad de transmitir conocimientos de una forma directa, práctica y actual, lo cual proporcionó a los autores las claves para desarrollar una metodología innovadora que recogiera dichas necesidades y despertara la motivación del alumnado. La innovación docente de esta actividad, enmarcada en el campo de la planificación del riego, consiste en desarrollar una serie de tareas que implica al alumno en la toma de decisiones con información en tiempo real. Abordar un cálculo de riego determinado no es problema nuevo, pero sí el hecho de abordarlo de la forma propuesta. El alumno parte de una serie de datos complejos que puede obtener de forma sencilla. Posteriormente es guiado en el análisis y las conclusiones son obtenidas de manera común junto a sus compañeros y profesores. Debido al conocimiento avanzado que se requiere para el manejo de esta información y su procesado, la innovación propuesta ha sido dirigida a alumnos del Máster en Ingeniería Agronómica, en la asignatua "Nuevas Tecnologías en Ingeniería Rural". Por otro lado es recomendable desarrollar la actividad en sesiones con 15 alumnos divididas en equipos de 3 componentes para favorecer el trabajo en grupo y la interacción con el profesor.

La metodología propuesta consta de tres partes de adquisición de datos y análisis de los mismos, y una cuarta que se centra en la evaluación y refuerzo de la propia actividad, que a su vez, será el producto de toda la tarea: el cálculo de tiempo de riego. Todas las partes mantienen un vinculo profesor-alumno activo y necesario.

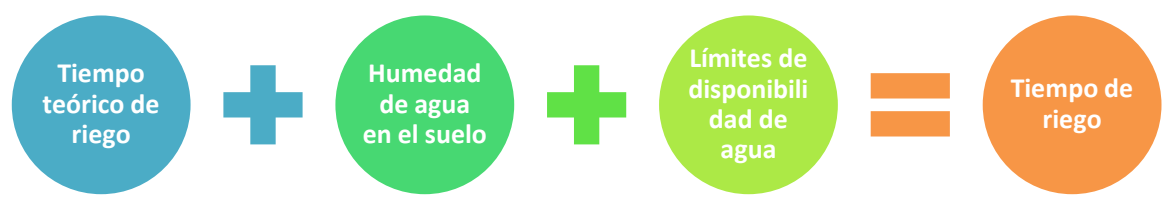

Fig. 2 Diagrama de tareas que componen la metodología propuesta.

A modo de introducción de los siguientes puntos donde se desarrollan las tareas de la metodología, y en concordancia con el diagrama de la Fig. 2, se presenta una tabla con los tiempos, medios necesarios y carácter presencial o no de las actividades. 
Tabla 1. Planificación tareas.

\begin{tabular}{|l|l|l|l|}
\hline Tareas & Tiempo & Medios a utilizar & Presencialidad \\
\hline Tiempo teórico & $45 \mathrm{~min}$ & Aula informática & Sí \\
\hline Humedad & $45 \mathrm{~min}$ & Aula informática & Sí \\
\hline Límites & $30 \mathrm{~min}$ & Dispositivo con Internet & No \\
\hline Tiempo riego & $90 \mathrm{~min}$ & Dispositivo con Internet y hoja de Excel & No \\
\hline
\end{tabular}

\subsection{Cálculo de las necesidades teóricas de riego}

El procedimiento para el cálculo de los volúmenes y/o tiempos de riego para un cultivo concreto es un procedimiento que acarrea dificultad y, en cierta manera, es ineficiente si no se utilizan medios informáticos. El principal objetivo de esta primera tarea es promover el uso de la plataforma pública y gratuita que proporciona el Servicio de Tecnología del Riego del Instituto Valenciano de Investigaciones Agrarias (STR - IVIA). Este centro es consciente de la importancia del servicio que ofrecen y por ello, realizan cursos y publicaciones en revistas divulgativas dentro del ámbito agrario (Bonet y Jordà, 2013; Jordà et al 2013). Concretamente, se trata de un servicio web (http://riegos.ivia.es/necesidadesde-riego) que permite obtener las necesidades de riego en base a las características básicas de la parcela y el cultivo, utilizando los datos meteorológicos recogidos en las diversas estaciones meteorológicas a las cuales tiene acceso este ente público. Dichas estaciones pertenecen al Sistema de Información Agroclimática para el Regadío - SIAR (http://eportal.magrama.gob.es/websiar/Inicio.aspx). 


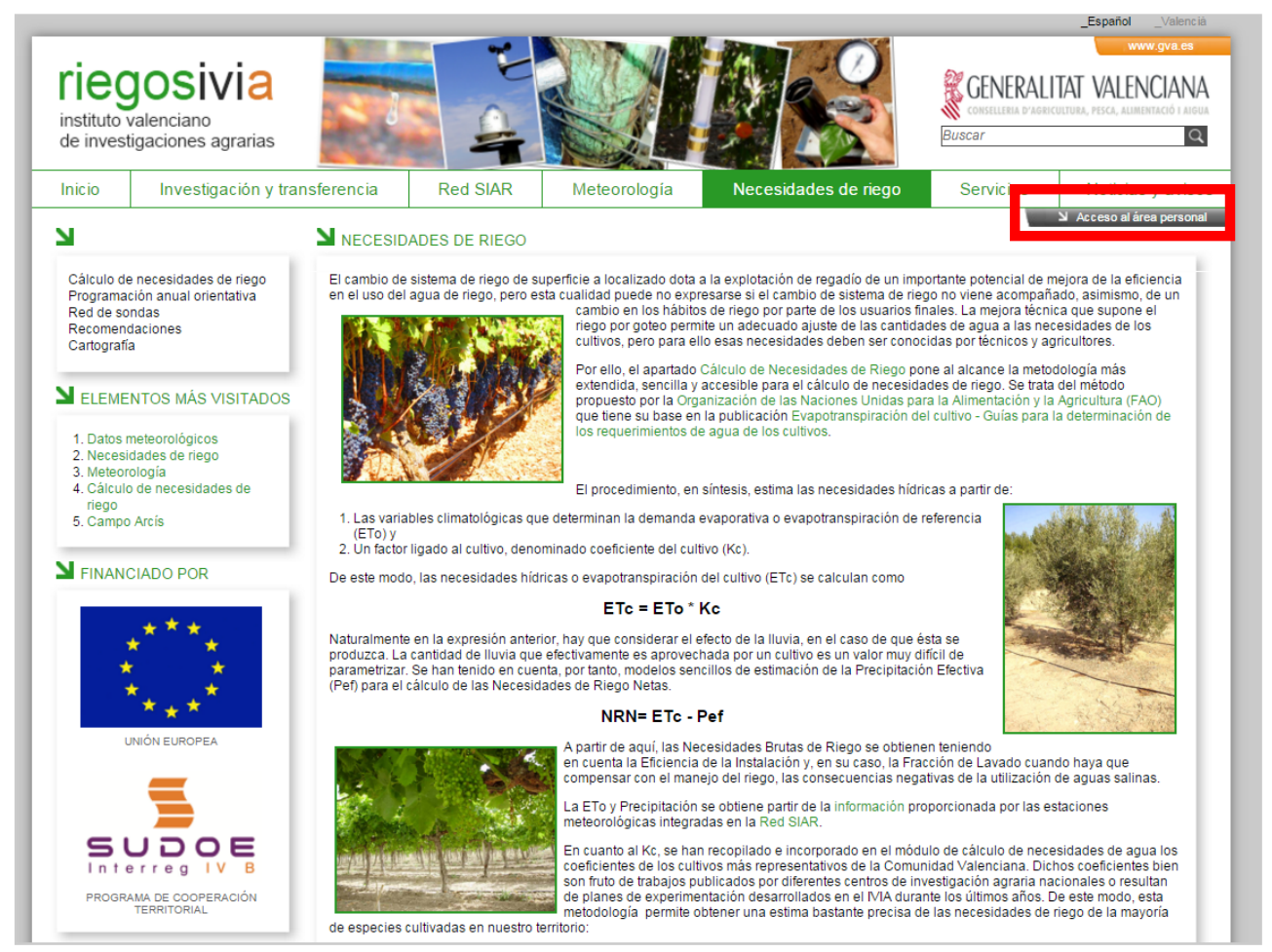

Fig. 3 Pantalla de inicio de la web del STR-IVIA. Destacado el enlace al acceso personal.

Con este procedimiento se ha pretendido hacer más dinámico y real el mencionado cálculo. Para poder utilizar la aplicación, el alumno debe darse de alta mediante el "Acceso al área personal" de forma y manera que podrá utilizar el servicio las veces que desee, creando un vínculo que le da continuidad en el tiempo a esta parte de la metodología. El alumno utiliza en el portal web datos obtenidos previamente en una sesión práctica desarrollada una parcela piloto, con el fin de familiarizarse con los conceptos requeridos de una forma más directa. Cabe destacar que este es un servicio que tiene multitud de opciones que vienen acompañadas de manuales en los que el alumno puede profundizar en función de sus necesidades intrínsecas.

Por otro lado, no es imprescindible el empleo de este servicio web, pudiendo diseñarse una herramienta alternativa sobre una hoja de cálculo convencional, pero las ventajas formativas son importantes: integra y actualiza información climática registrada diariamente de múltiples localizaciones de manera dinámica, incorpora a través de formularios variables agronómicas, es una herramienta profesional contrastada por técnicos e investigadores y enlaza información que permite el autoaprendizaje y el estudio de casos específicos.

\subsection{Descripción y análisis del dispositivo de medida de humedad del suelo}

La segunda parte de la metodología de trabajo es una de las más innovadoras a la hora de transmitir nuevos conocimientos por la aplicabilidad y potencial de la misma. Consiste en manejar los conceptos fundamentales de un tipo de equipos que miden el contenido 
volumétrico de agua en el suelo en una parcela determinada. Concretamente se propone trabajar con las sondas denominadas Frequency Domain Reflectometry (FDR) que miden el nivel de humedad indirectamente a partir de la medición de la constante dieléctrica aparente del suelo. Las sondas se fijan en el terreno y están conectadas a un registrador de datos que envía la información vía Internet hasta un servidor determinado, de forma y manera que se conoce la disponibilidad de agua en el suelo en tiempo real. Véase en la Fig. 4, a modo de ejemplo, un esquema del dispositivo y su ubicación.

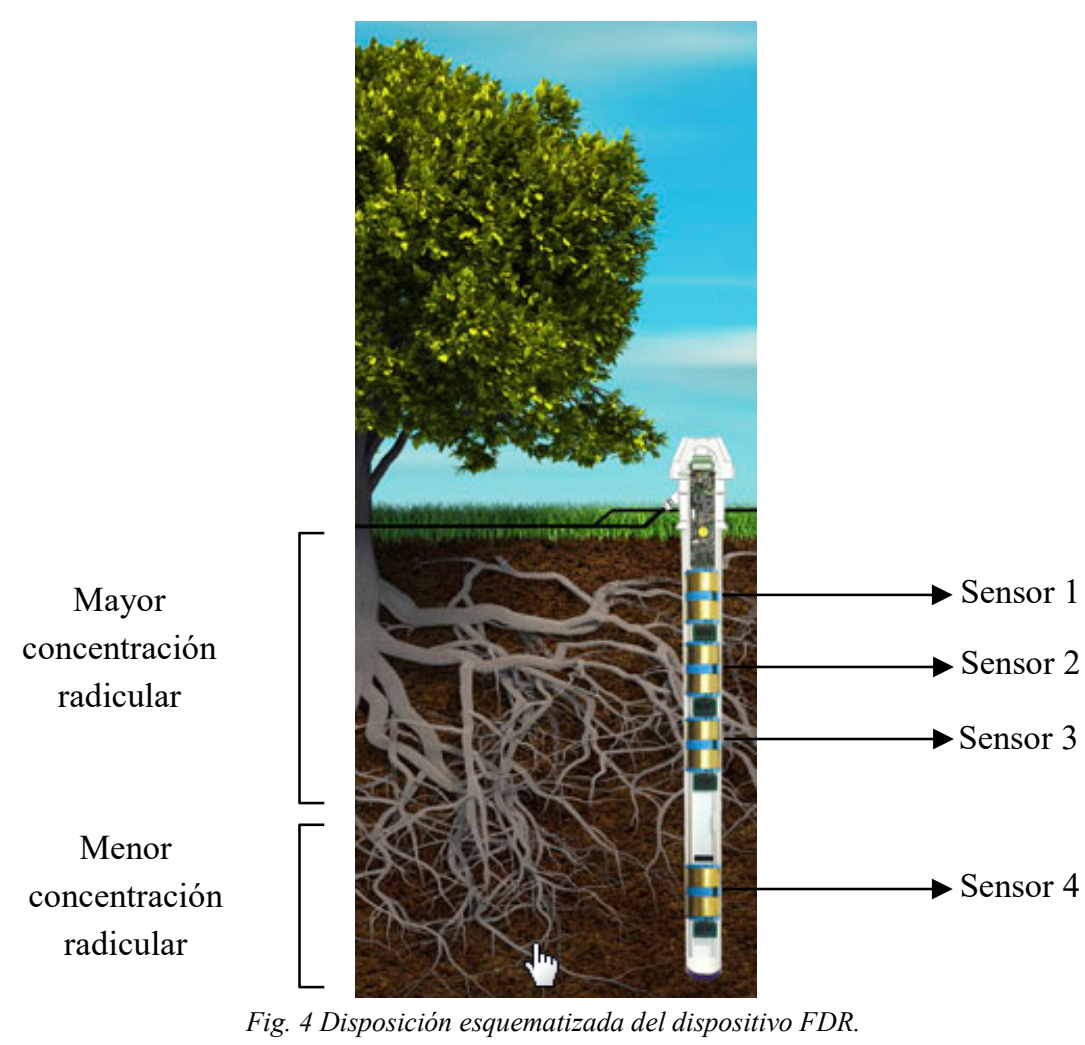

Dependiendo del cultivo, los sensores de medida se disponen a distintas profundidades para monitorizar el contenido de agua en las dos principales áreas diferenciadas de la zona radicular, la de mayor y menor contenido de raíces. Estos sensores proporcionan una información muy valiosa que debe ser evaluada desde diferentes puntos de vista relacionados con las siguientes etapas de la metodología. En esta fase se revisan y refuerzan conceptos de medición de parámetros físicos e instrumentación, adquisición y almacenamiento de datos y comunicación a distancia con diferentes tecnologías.

\subsection{Determinación de las características hidráulicas del suelo}

La introducción de nuevas bases de datos para el cálculo de las necesidades teóricas del riego así como el estudio de los equipos de medida de humedad del suelo, novedosos para el alumnado, no dejan de lado los estudios desarrollados en las clases teóricas ya que permiten interpretar la información que nos proporcionan dichos dispositivos. Se debe

(cc) EY-NC-ND 2016, Universitat Politècnica de València

Congreso In-Red (2016) 
revisar el concepto de curva característica de humedad que viene a ser la característica principal del suelo en relación con su capacidad de retención de agua (Ingelmo y Cuadrado, 1986). De esta forma se definen dos puntos límite entre los cuales debe situarse el contenido de agua en el suelo medido por las sondas FDR. Concretamente se habla de la capacidad de campo (CC) que coincide con la cantidad máxima de agua que el suelo puede retener sin drenaje en profundidad, y el punto de marchitez permanente (PMP) que es el contenido de humedad del suelo por debajo del cual las plantas no pueden extraer agua para cubrir sus necesidades.. En el siguiente gráfico se puede ver un ejemplo de dichos puntos y la curva que los representa.

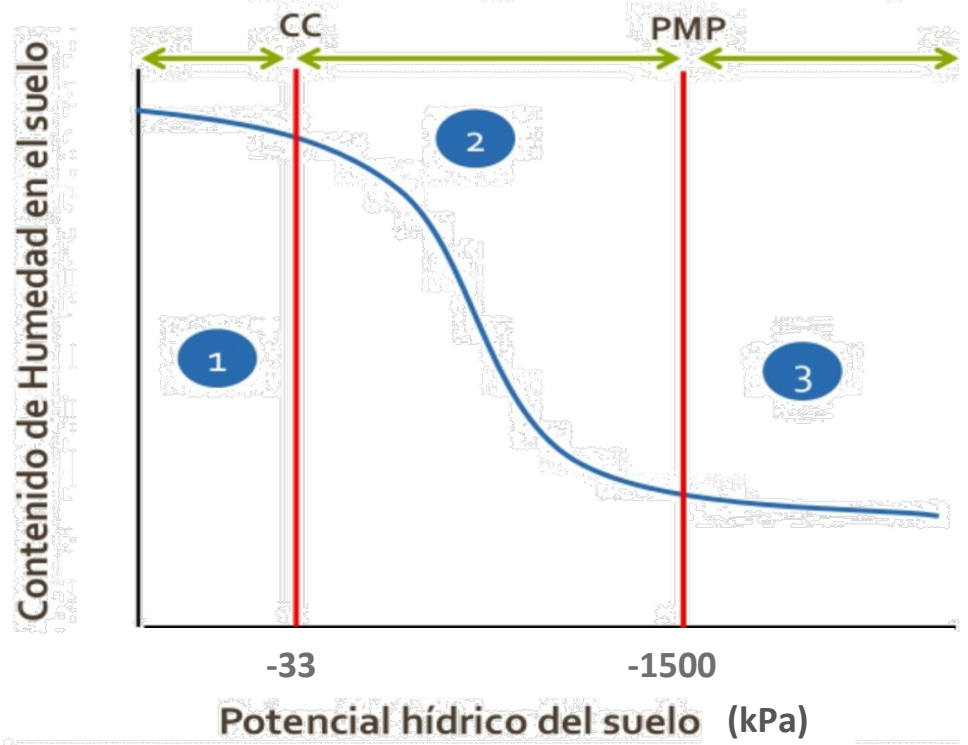

Fig. 5 Curva característica de humedad. Se destaca el agua gravitacional que se pierde por percolación (1), el agua capilar o aprovechable (2) y el agua higroscópica o no disponible (3).

Esta curva de vital importancia para la interpretación de los datos de la sonda FDR y la caracterización de la calidad del riego, se obtiene mediante la utilización de las denominadas placas de Richard (cámaras de presión). La utilización de dichos equipos se lleva a cabo mediante una explicación teórica y la visualización de videos disponibles en YouTube (Labferrer, 2016; Politube 2016), debido a que la duración del ensayo puede llegar a ocupar varias semanas. Por lo tanto, los puntos de CC y PMP son proporcionados el profesor. Los conceptos teóricos incorporados en esta fase se asientan en las bases de la agronomía y la ciencia del suelo, son recuperados para un uso práctico en la gestión del riego; es muy adecuada su síntesis en videos.

\subsection{Interpretación y evaluación de resultados..}

La información obtenida en las anteriores etapas de la metodología se integra de la siguiente forma:

- Obtener el dato de tiempo y/o volumen de agua teórico mediante el servicio web.

- Descargar del servidor de almacenamiento y representar gráficamente los valores proporcionados por la sonda FDR. 
- Incluir en la gráfica de humedad los límites de CC y PMP.

Una vez obtenidas la gráfica con la evolución de la humedad del suelo y las recomendaciones de riego (recomendable utilizar datos de una semana previa a la realización de la actividad), se deben tener en cuenta cuatro características básicas que permiten modificar, tanto al alza como a la baja, el valor teórico del riego:

- Los sensores 1 a 3 referidos en la Figura 4 deben registrar cada pulso de riego, es decir, se debe detectar una elevación y posterior disminución del nivel de humedad, de forma y manera que el agua de riego alcance la zona de mayor concentración de raíces.

- Los sensores 1 a 3 referidos en la Figura 4 deben mantener sus niveles dentro del rango determinado como CC y PMP, pues el agua estará a disposición de la planta sin exceso ni defecto.

- Los sensores 1 a 3 referidos en la Figura 4 han de mostrar una disminución a lo largo del período de estudio del contenido de agua en el suelo moderado, con pendientes suaves que eviten tener que realizar riegos de altos volúmenes en períodos cortos.

- El sensor 4 referido en la Figura 4 no debe registrar los pulsos de riego, pues se localiza en la zona de menor concentración de raíces y sería sinónimo de pérdidas por percolación profunda.

\section{Ejemplo de aplicación y resultados}

Este planteamiento innovador que destaca la toma de decisiones en tiempo real, requiere resolver cada uno de los apartados previamente descritos. Tal y como se indica en la introducción, es una actividad que fomenta la autonomía del alumno, y a su vez el trabajo en equipo.

Al iniciar un ejemplo clásico, en primer lugar se debe definir una parcela y un periodo de tiempo para el que se propondrá una programación de riego (dosis de agua, duración de los riegos e intervalo entre los mismos) basando el cálculo en muchas ocasiones en datos estimados o promediados. Aquí se ha contado con una parcela con instrumentación, de la que se conoce en cualquier instante el contenido de humedad del suelo a diferentes profundidades, así como datos climáticos (o su pronóstico en su caso) del periodo de programación.

El primer aparatado que se refiere a utilizar el servicio de cálculo de necesidades de riego del STR - IVIA, obligaba al alumno a trabajar con datos reales que identifique el cultivo de estudio. Concretamente se detalló el tipo de cultivo (en un desplegable de diversas opciones), el diámetro de copa, el marco de plantación, el número de emisores por planta y el caudal unitario de los mencionados emisores. Existen otras opciones adicionales, como es el dato de salinidad de agua, que es interesante incluir pues apoyan los conceptos desarrollados en las clases teóricas. En la Fig. 5 se pueden ver las diferentes opciones a introducir, incluidos los datos meteorológicos de la estación más próxima a la parcela piloto.

(cc) EY-NC-ND 2016, Universitat Politècnica de València

Congreso In-Red (2016) 


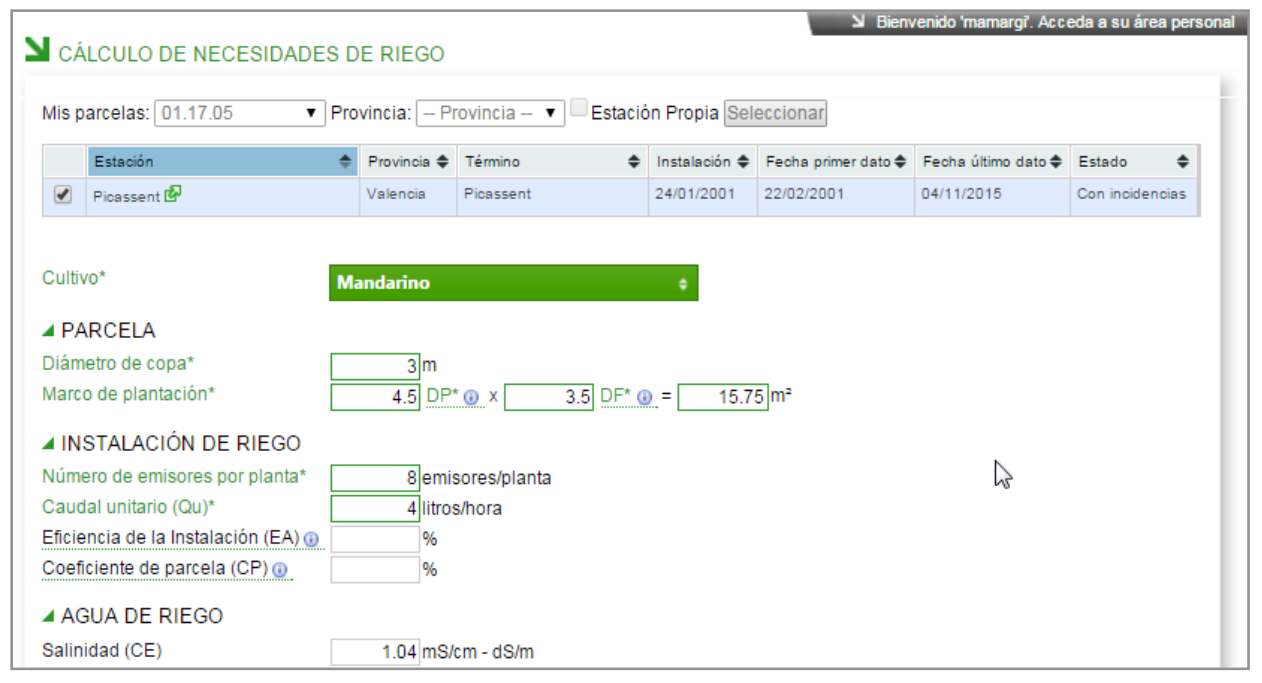

Fig. 5 Principales datos de entrada para el cálculo teórico de las necesidades de riego

Adicionalmente, existe un apartado en la misma página (Fig. 6) que permite seleccionar el rango de tiempo para el cálculo, así como la posibilidad de incluir la precipitación registrada y finalmente realizar el cálculo. El resultado que proporciona el servidor es un documento Excel con la información del tiempo de riego teórico. Este valor es la referencia que luego se trabajó con los resultados de las sondas.

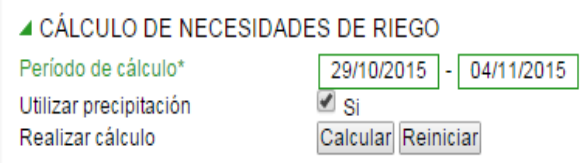

Fig. 6 Principales datos de entrada para el cálculo teórico de las necesidades de riego

Posteriormente, y una vez proporcionados por el profesor los valores de CC y PMP, se presentó el ejemplo de la Fig. 7 para que el alumno pudiera construir unas gráficas análogas para su posterior evaluación, correspondientes a los registros de la sonda FDR de la semana previa al cálculo de la recomendación. Con estos datos, se analizaron las cuatro tendencias descritas en el apartado 3.4. para caracterizar el riego. En este ejemplo concreto se presentó un registro en el que se detectan todos los puntos críticos descritos, que obligarían a modificar el tiempo de riego teórico con el fin de obtener una evolución de la humedad del suelo dentro de los parámetros considerados como adecuados. 

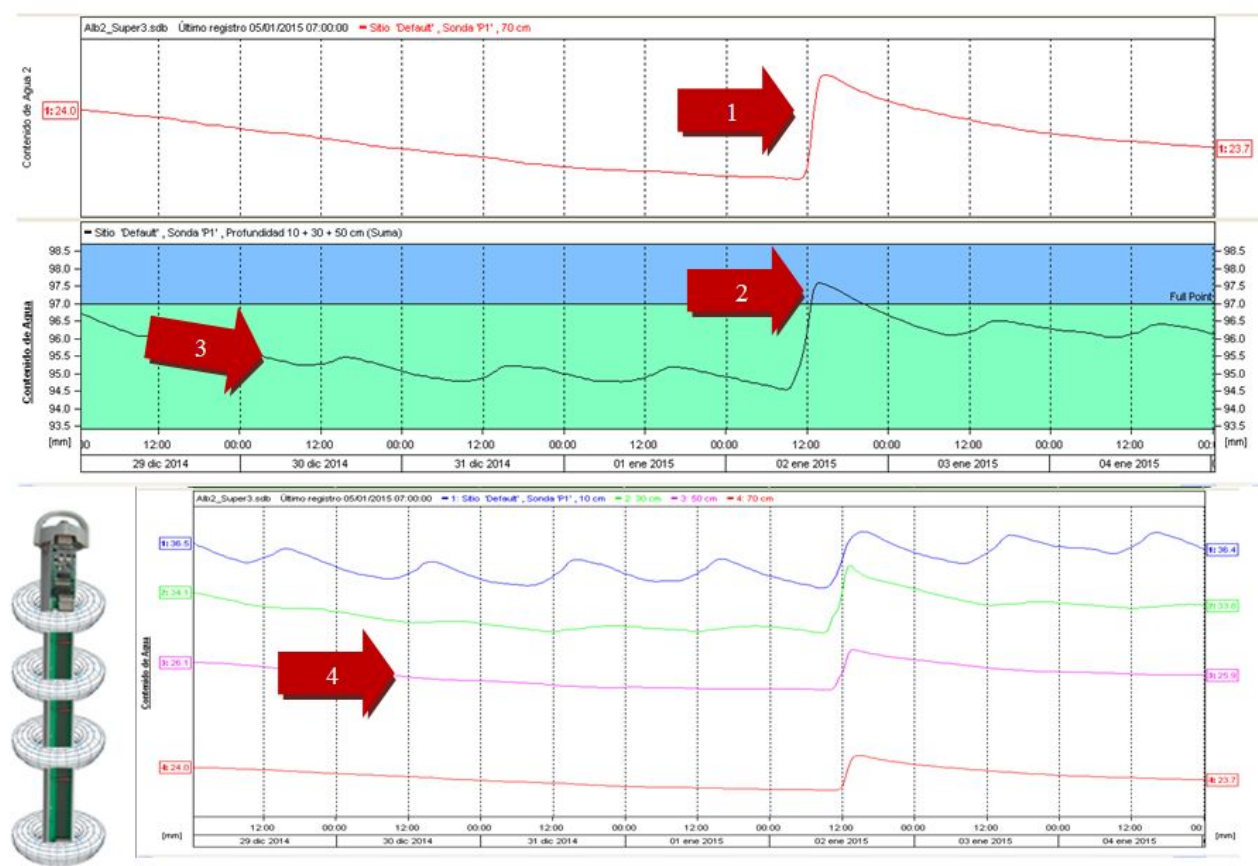

Fig. 7 Gráficas de evolución del contenido del agua en el suelo. Líneas en azul, verde, rosa y rojo, sensores a 10, 30, 50 y $70 \mathrm{~cm}$ (sensores 1, 2, 3 y 4) respectivamente. Linea negra, suma sensores a 10, 30 y $50 \mathrm{~cm}$. Área en verde delimitada por la CC y el PMP. Flechas en rojo indicativas de puntos críticos.

Concretamente se detectan los siguientes puntos críticos:

1. Se observa que el sensor localizado a $70 \mathrm{~cm}$ (sensor 4) detecta un incremento del contenido de agua en el suelo, considerando que el caso de estudio son cítricos, este punto indicaría que está recibiendo agua una zona con poca concentración de raíces y posiblemente se esté perdiendo agua por percolación profunda.

2. Ese mismo punto es coincidente con la excesiva elevación de la gráfica delimitada entre las áreas azul (sensor 1) y verde (sensor 2), indicativo de que se ha superado la CC y por tanto se está perdiendo agua porque el suelo está saturado.

3. También se observa la excesiva pendiente con la que disminuye el contenido de agua.

4. Finalmente, se detecta que no todos los sensores registran un aumento de humedad del suelo en cada pulso de riego. Únicamente el sensor de $10 \mathrm{~cm}$ (el más superficial, sensor 1) tiene un comportamiento adecuado.

De este análisis se pudieron obtener las siguientes conclusiones: i) el riego es ineficiente porque hay pérdidas en profundidad que el cultivo no utiliza; ii) no todos los sensores localizados en la zona de mayor concentración radicular detectan el riego; y iii) la disminución del contenido en el suelo es demasiado rápida llegando a valores cercano al PMP. Con todo ello, se recomendó aumentar el tiempo de riego teórico fraccionando en dos riegos al día para mejorar la retención y uso del agua por parte de la planta. 
Es importante resaltar que esta recomendación de riego a partir del tiempo teórico y el aumento y fraccionamiento del tiempo de riego, es un resultado interpretable que posiblemente no será coincidente con el óptimo, pero será adecuado para mostrar cómo manejar recursos complejos de una forma eficiente.

La evaluación de los alumos, se realizó a partir de la ejecución de un ejemplo similar por parte de cada grupo. Incluyendo la descarga directa de datos de los servidores e interpretación de los resultados, para poder realizar una comparativa con los compañeros del grupo. Con todo ello, se indicó que se subiera un informe a la plataforma PoliformaT de la asignatura, de extensión máxima 3 hojas, con los resultados obtenidos por cada componente, las diferencias respecto a una recomendación final consensuada entre el grupo y una conclusión final de forma que se potenciara indirectamente la coevaluación. El trabajo se valoró desde dos puntos de vista, es decir, nivel de competencias específicas y transversales adquirido. Por una parte, el producto de la actividad se evaluó a tres niveles (aceptable, adecuado y erróneo). Por otra, el proceso de evaluación de las competencias transversales se realizó mediante rubrica considerando los niveles 2 y 3 adecuados a nivel de máster. Para todo ello, se emplearon los descriptores que permitieron profundizar en el nivel de los conocimientos adquiridos, la claridad del informe, la coherencia entre los tiempos de riego determinados, el grado de consenso alcanzado por los componentes del grupo, y finalmente, precisión de los resultados.

La evaluación de esta innovación fue realizada mendiante una encuesta sencilla a los alumnos, diseñada sobre la herramienta de formularios de Google que respondida al concluir la última operación sobre PoliformaT. Se incluían preguntas básicas para valorar la utilidad e interés profesional y académico, así como la claridad y organización de las distintas fases de la práctica. Cada pregunta se respondía a cino niveles, mas la opción de incluir comentarios, sugrencias o posibles mejoras. La respuesta por parte de los alumnos fue satisfactoria, destacándose el interés práctico; como aspectos a mejorar en su valoración cabe mencionar una mejor distribución de los tiempos de cada fase

\section{Conclusiones}

Esta actividad incluye un el uso de un servicio web público y gratuito que debería ser conocido por el conjunto de alumnos a los que va dirigida la actividad, así mismo se introduce el uso de una tecnología innovadora en el ámbito del riego y sobre todo el uso que se hace de la misma, la obtención vía remota de datos complejos de forma sencilla y en tiempo real. Ambos componentes llevan a trabajar los conceptos teóricos desarrollados en clase desde un punto de vista práctico y de aplicación directa. Son múltiples las comunidades de regantes y técnicos que hoy día emplean este enfoque para el cálculo de las necesidades de riego, siendo una tendencia creciente con el fin de optimizar el uso de los recursos. Es una actividad que acerca al alumno a la realidad, recapitulando conceptos básicos de la tecnología del riego, analizando situaciones reales y potenciando la autonomía y el consenso desde la búsqueda de soluciones coherentes.

(c)) BY-NC-ND 2016, Universitat Politècnica de València 
Esta actividad requiere de una completa coordinación entre el profesorado que desarrolle las clases teóricas y las prácticas de campo, laboratorio e informática; pero los resultados incipientes obtenidos sobre el grupo procedente de Chile y con los alumnos de Máster, muestran que el interés sobre este tipo de metodologías es significativo, lo que supone un mayor éxito de la transferencia del conocimiento dentro del diverso grupo de alumnos que compone las aulas.

\title{
6. Referencias
}

BAIN, K. (2006). Lo que hacen los mejores profesores de universidad. Valencia. Universitat de València.

BONET, L. y JORDÀ, M. (2013). "El nuevo portal de riegos del Instituto Valenciano de Investigaciones Agrarias ". Agrícola vergel: Fruticultura, horticultura, floricultura. 32(369), 333-336.

FAO - AQUASTAT website (2016). Food and Agriculture Organization of the United Nations (FAO). Proportion of total water withdrawal withdrawn for agriculture (\%). $<\underline{\text { http://www.fao.org/nr/water/aquastat/maps/World-Map.WithA.Twith eng.htm }>}$ [Consulta: 30 de marzo de 2016]

INGELMO SÁNCHEZ, F y CUADRADO SÁNCHEZ, S. (1986). "El agua en el suelo" en Ingelmo Sánchez, F y Cuadrado Sánchez, S. El agua y el medio fisico del suelo. Salamanca. Europa Artes Gráficas S.A. Centro de Edafología y Biología Aplicada (CSIC). Excma. Diputación Provincial.

INSTITUTO DE CIENCIAS DE LA EDUCACIÓN. Competencias transversales UPV. $<$ http://www.upv.es/entidades/ICE/info/931816normalc.html $>$ [Consulta 21 de marzo de 2016]

JORDÀ, M., INRIGLIOLO, D.S. y BONET, L. (2013). "Mejora de la eficiencia en el riego en la Comunitat Valenciana: El portal de riegos del Instituto Valenciano de Investigaciones Agrarias". Agricultura y cooperación, $N^{o} 337$

LAB_FERRER Métodos avanzados para determinar la Capacidad de Campo del suelo. Youtube $<$ https://www.youtube.com/watch?v=o4XLmxlJijQ > [Consulta: 24 de Marzo de 2016]

POLITUBE. UNIVERSIDAD POLITÉCNICA DE VALENCIA. Determinación del estado hídrico

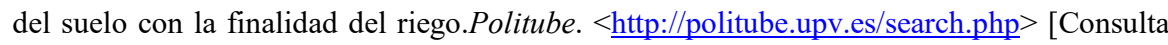
2 de marzo de 2016]

RIEGOS IVIA. INSTITUTO VALENCIANO DE INVESTIGACIONES AGRARIAS. Necesidades de riego. $<$ http://riegos.ivia.es/necesidades-de-riego $>$ [Consulta: 21 de marzo de 2016]

\author{
SISTEMA DE INFORMACION AGROCLIMATICA PARA EL REGADIO. MINISTERIO DE \\ AGRICULTURA, ALIMENTACION Y MEDIO AMBIENTE. \\ http://eportal.magrama.gob.es/websiar/Inicio.aspx [Consulta 16 de marzo de 2016]
}

2016, Universitat Politècnica de València

Congreso In-Red (2016) 